\section{Acceptance of two \\ oral formulations of ciclosporin in dogs}

\section{Kelly Doucette ${ }^{1}$, Srinivas Kammanadiminti ${ }^{1}$, Lori Carter ${ }^{2}$, Wolfgang Seewald ${ }^{1}$, Jerome Giraudel ${ }^{1}$}

1 Elanco Animal Health, Greenfield, Indiana, USA

2 Stillmeadow Inc., Sugar Land, Texas, USA

\section{OBJECTIVE}

Determine and compare the voluntary acceptance of two oral formulations of ciclosporin.

\section{MATERIALS \& METHODS}

Twenty-five adult mixed breed dogs in a cross-over design. Phase 1, consisted of dosing Group 1 (12 animals) with ciclosporin A oral solution, USP MODIFIED (Treatment A) for 7 days via oral syringe while Group II (13 animals) received a generic ciclosporin $A$ oral solution
(Treatment B) daily with cross-over after 3-day washout. In Phase 2 (period 1), after a 2 day washout, tested products, administered to dogs in same order as Phase I, were mixed in a small amount of food for 3 days, approximately 6 hours after morning feeding with food left in cage followed with cross-over after a 2-day washout. In period 2 of Phase 2, food was offered as in the first half of Phase 2, one hour in the morning and then withdrawn, with test article offered in food five hours later with similar cross-over of treatments.

\section{RESULTS}

Phase I had $100 \%$ voluntary acceptance for Treatment A, and $98.9 \%$ voluntary acceptance of Treatment B. Phase 2 (Period 1), had $61.1 \%$ immediate prehension of Treatment $A$ and $56.4 \%$ immediate prehension of Treatment B. During Phase 2 (Period 2), immediate prehension was $69.2 \%, 69.4 \%$ and $92.0 \%$ for Treatment A, Treatment B and the positive control (high calorie diet supplement), respectively. Two adverse events (diarrhea and vomiting) had a probable relationship to the test articles.

\section{CONCLUSION}

No significant differences in the acceptance of the two oral ciclosporin solutions for dogs.

\section{Canine}

pseudopregnancy: an evaluation of incidence, diagnosis and current treatment protocols

\section{Amanda Root ${ }^{1}$, Tim Parkin ${ }^{1}$, Pippa Hutchison ${ }^{1}$, Caroline Warnes ${ }^{2}$, Philippa Yam ${ }^{1}$}

1 University of Glasgow, Glasgow, Scotland, UK

2 Good Companions, Swindon, England, UK

\section{OBJECTIVES}

To assess how often vets recognise cases of pseudopregnancy in bitches, which clinical and/or behavioural signs are used for diagnosis, and which management or treatment protocols are used.

\section{METHODS}

A postal questionnaire on pseudopregnancy was sent to 2000 practising veterinary surgeons in 2015 . Descriptive statistics were obtained, prevalence of outcomes estimated and $95 \%$ confidence intervals calculated.

\section{RESULTS}

The response rate was $19.8 \%$. Clinical pseudopregnancy has been estimated in previous literature to be as high as $50 \%$ in the domestic dog. Veterinary surgeons reported that $46 \%$ of bitches showed behavioural signs of pseudopregnancy without any physical signs and collecting/ mothering objects was the most frequent (96\%). Sixtythree percent of vets had seen aggression in pseudopregnant bitches. Nevertheless, only $52 \%$ of vets routinely asked owners about behaviour changes during consultations. Eight percent of respondents reported seeing pseudopregnancy in spayed bitches. The most common reported clinical sign was enlarged mammary glands and/or milk production (89\%). Treatment options varied (surgical, medical or none) and depended on duration and severity of clinical and behavioural signs, owners' preference, cost, concurrent disease, drug availability and previous history.

\section{STATEMENT}

This is the largest epidemiological study of canine pseudopregnancy in the UK. Progress on our understanding of diagnosis and treatment of pseudopregnancy in spayed and entire bitches has been made. The prevalence and severity of clinical and behavioural signs in dogs with pseudopregnancy are variable and possibly under-estimated. Dogs with overt pseudopregnancy experience diverse clinical and behavioural problems and standard treatment protocols are lacking. 